PROCEEDINGS OF THE

AMERICAN MATHEMATICAL SOCIETY

Volume 138, Number 7, July 2010, Pages 2431-2441

S 0002-9939(10)10267-6

Article electronically published on March 24, 2010

\title{
BURKHOLDER'S INEQUALITIES IN NONCOMMUTATIVE LORENTZ SPACES
}

\author{
YONG JIAO
}

(Communicated by Marius Junge)

\begin{abstract}
We prove Burkholder's inequalities in noncommutative Lorentz spaces $L^{p, q}(\mathcal{M}), 1<p<\infty, 1 \leq q<\infty$, associated with a von Neumann algebra $\mathcal{M}$ equipped with a faithful normal tracial state. These estimates generalize the classical inequalities in the commutative case.
\end{abstract}

\section{INTRODUCTION}

Martingale inequalities and sums of independent random variables are important tools in classical harmonic analysis. A fundamental result due to Burkholder 1, 2 can be stated as follows. Given a probability space $(\Omega, \mathscr{F}, P)$, let $\left\{\mathscr{F}_{n}\right\}_{n>1}$ be a nondecreasing sequence of $\sigma$-fields of $\mathscr{F}$ such that $\mathscr{F}=\vee \mathscr{F}_{n}$ and let $\mathbb{E}_{n}$ be the conditional expectation operator relative to $\mathscr{F}_{n}$. Given $2 \leq p<\infty$ and an $L^{p}$-bounded martingale $f=\left(f_{n}\right)_{n \geq 1}$, we have

$$
\|f\|_{L^{p}} \approx\left\|\left(\sum_{k=1}^{\infty} \mathbb{E}_{k-1}\left(\left|d f_{k}\right|^{2}\right)\right)^{1 / 2}\right\|_{L^{p}}+\left\|\left(\sum_{k=1}^{\infty}\left|d f_{k}\right|^{p}\right)^{1 / p}\right\|_{L^{p}} .
$$

The first term on the right is called the conditioned square function of $f$, while the second is called the $p$-variation of $f$. Rosenthal's inequalities 14 can be regarded as the particular case where the sequence $d f=\left(d f_{1}, d f_{2}, \ldots\right)$ is a family of independent mean-zero random variables $d f_{k}=a_{k}$. In this case it is easy to reduce Rosenthal's inequalities to

$$
\left\|\sum_{k=1}^{\infty} a_{k}\right\|_{L^{p}} \approx\left(\sum_{k=1}^{\infty}\left\|a_{k}\right\|_{2}^{2}\right)^{1 / 2}+\left(\sum_{k=1}^{\infty}\left\|a_{k}\right\|_{p}^{p}\right)^{1 / p}
$$

Noncommutative analogues of the above inequalities were successfully obtained by Junge and $\mathrm{Xu}$ in [10] and [11]. They replaced conditioned expectations onto the $\sigma$ subfields by conditioned expectations onto an increasing sequence of von Neumann subalgebras of a given von Neumann algebra. More precisely, for $2 \leq p<\infty$ and

Received by the editors January 13, 2009, and, in revised form, September 22, 2009.

2000 Mathematics Subject Classification. Primary 46L53; Secondary 60G42.

Key words and phrases. Noncommutative martingales, Burkholder's inequalities, Lorentz spaces.

The author was partially supported by the Agence Nationale de Recherche (06-BLAN-0015), the National Natural Science Foundation of China (10671147) and the China Scholarship Council (2007U13085).

(C)2010 American Mathematical Society Reverts to public domain 28 years from publication 
any finite noncommutative $L^{p}(\mathcal{M})$-martingale $x=\left(x_{n}\right)_{n \geq 1},(1.1)$ has the following noncommutative version:

$$
\|x\|_{L^{p}(\mathcal{M})} \approx \max \left\{\left\|\left(\sum_{k}\left|d x_{k}\right|^{p}\right)^{1 / p}\right\|_{L^{p}(\mathcal{M})},\left\|s_{c}(x)\right\|_{L^{p}(\mathcal{M})},\left\|s_{r}(x)\right\|_{L^{p}(\mathcal{M})}\right\},
$$

where $s_{c}(x)$ and $s_{r}(x)$ denote column and row versions of the conditioned square function. Moreover, they obtained a simpler inequality for $1<p \leq 2$ by duality. Recently, Randrianantoanina [15] proved a weak-type inequality for conditioned square functions, which implies Junge and Xu's noncommutative Burkholder inequalities by interpolation. This alternate approach yields better constants, some of which are optimal.

Our original motivation comes from the classical extension to Lorentz spaces of Rosenthal's inequalities (1.2) by Carothers and Dilworth [5], i.e., for $2<p<$ $\infty, 0<q \leq \infty$, and any independent mean-zero random variables $f_{1}, f_{2}, \ldots, f_{n}$,

$$
\left\|\sum_{k=1}^{n} f_{k}\right\|_{L^{p, q}(\Omega)} \approx \max \left\{\left\|\sum_{k=1}^{n} f_{k}\right\|_{L^{2}(\Omega)},\left\|\sum_{k=1}^{n} \oplus f_{k}\right\|_{L^{p, q}(0, \infty)}\right\},
$$

where $\sum_{k=1}^{n} \oplus f_{k}$ denotes the disjoint sum of $f_{1}, f_{2}, \ldots, f_{n}$, which is a function on $(0, \infty)$ with $d_{f}(t)=\sum_{k=1}^{n} d_{f_{k}}(t)$.

Inspired by (1.3) and (1.4), in this paper we consider Burkholder's inequalities in noncommutative Lorentz spaces $L^{p, q}(\mathcal{M}), 1<p<\infty, 1 \leq q<\infty$, and one of our main results can be stated as follows (see Theorem 3.1 for the detailed statement): for $2<p<\infty, 1 \leq q<\infty$, and any finite $L^{p, q}(\mathcal{M})$-martingale $x$, we have

$$
\|x\|_{L^{p, q}(\mathcal{M})} \approx \max \left\{\left\|\sum_{k} d x_{k} \otimes e_{k}\right\|_{L^{p, q}\left(\mathcal{M} \otimes \ell^{\infty}\right)},\left\|s_{c}(x)\right\|_{L^{p, q}(\mathcal{M})},\left\|s_{r}(x)\right\|_{L^{p, q}(\mathcal{M})}\right\} .
$$

Note that if $p=q$, we come back to the inequalities (1.3). We also extend these inequalities to the case where $1<p<2,1 \leq q<\infty$. Our main results are contained in section 3. Note that the proofs of these inequalities for $L^{p}$-spaces in [10] and [11 use an iteration argument; however, this iteration seems inefficient (or more complicated) for the case of Lorentz spaces. We will adopt a different approach based on the Randrianantoanina weak-type $(1,1)$ inequality.

\section{Preliminaries}

Let $(\Omega, \mathscr{F}, P)$ be a (commutative) probability space and $f$ a random variable on $(\Omega, \mathscr{F}, P)$. The decreasing rearrangement of $f$, denoted by $f^{*}$, is $f^{*}(t)=\inf \{s>0$ : $P(|f|>s) \leq t\}, t \in[0,1]$. The Lorentz space $L^{p, q}(\Omega)=L^{p, q}, 0<p<\infty, 0<q \leq$ $\infty$, consists of those measurable functions with finite quasi-norm $\|f\|_{p, q}$ given by

$$
\begin{gathered}
\|f\|_{p, q}=\left(\int_{0}^{\infty}\left(t^{1 / p} f^{*}(t)\right)^{q} \frac{d t}{t}\right)^{1 / q}, \quad 0<q<\infty, \\
\|f\|_{p, \infty}=\sup _{t>0} t^{1 / p} f^{*}(t), \quad q=\infty .
\end{gathered}
$$

It is well known that if $1<p<\infty$ and $1 \leq q \leq \infty$, then $\|f\|_{p, q}$ is equivalent to a norm. Hölder's inequality for Lorentz spaces is the following, which first appeared in the work of O'Neil [8]:

$$
\|f g\|_{p, q} \leq C\|f\|_{p_{0}, q_{0}}\|g\|_{p_{1}, q_{1}}
$$


for all $0<p, q, p_{0}, q_{0}, p_{1}, q_{1} \leq \infty$ such that $1 / p=1 / p_{0}+1 / p_{1}$ and $1 / q=1 / q_{0}+1 / q_{1}$. We refer to 3], 4, and [7] for details on classical (commutative) Lorentz spaces.

Now we introduce the noncommutative Lorentz spaces. Let $(\mathcal{M}, \tau)$ be a tracial noncommutative probability space. Namely, $\mathcal{M}$ is a von Neumann algebra with a normal faithful normalized trace $\tau$. We refer to 13 . for noncommutative integration and additional historical references. We only briefly recall some elementary facts about noncommutative Lorentz spaces. Let $L_{0}(\mathcal{M})$ denote the topological $*$-algebra of all measurable operators with respect to $(\mathcal{M}, \tau)$. For $x \in L_{0}(\mathcal{M})$, define its generalized singular number by

$$
\mu_{t}(x)=\inf \left\{\lambda>0: \tau\left(\mathbf{1}_{(\lambda, \infty)}(|x|)\right) \leq t\right\}, \quad t>0 .
$$

Then for $0<p<\infty$,

$$
L^{p}(\mathcal{M})=\left\{x \in L_{0}(\mathcal{M}): \tau\left(|x|^{p}\right)<\infty\right\}
$$

and

$$
\|x\|_{L^{p}(\mathcal{M})}^{p}=\tau\left(|x|^{p}\right)=\int_{0}^{\infty}\left(\mu_{t}(t)\right)^{p} d t .
$$

Of special interest in this paper are the noncommutative Lorentz spaces $L^{p, q}(\mathcal{M})$ associated with $(\mathcal{M}, \tau)$ :

$$
L^{p, q}(\mathcal{M})=\left\{x \in L_{0}(\mathcal{M}):\|x\|_{L^{p, q}(\mathcal{M})}<\infty\right\}
$$

where

$$
\|x\|_{L^{p, q}(\mathcal{M})}=\left(\int_{0}^{\infty}\left(t^{1 / p} \mu_{t}(x)\right)^{q} \frac{d t}{t}\right)^{1 / q}
$$

for $0<q<\infty$ and with the usual modification for $q=\infty$.

The noncommutative Lorentz spaces behave well with respect to real interpolation. Let $0<\theta<1,0<p_{k}, q_{k} \leq \infty$ for $k=0,1$ and $p_{0} \neq p_{1}$. Then

$$
L^{p, q}(\mathcal{M})=\left[L^{p_{0}, q_{0}}(\mathcal{M}), L^{p_{1}, q_{1}}(\mathcal{M})\right]_{\theta, q}
$$

where $1 / p=(1-\theta) / p_{0}+\theta / p_{1}, 0<q \leq \infty$.

The usual Hölder inequality also extends to the noncommutative setting. Let $0<p_{k}, q_{k} \leq \infty$ for $k=0,1$ and $1 / p=1 / p_{0}+1 / p_{1}, 1 / q=1 / q_{0}+1 / q_{1}$. Then for any $x \in L^{p_{0}, q_{0}}(\mathcal{M})$ and $y \in L^{p_{1}, q_{1}}(\mathcal{M})$,

$$
\|x y\|_{L^{p, q}(\mathcal{M})} \leq C\|x\|_{L^{p_{0}, q_{0}(\mathcal{M})}}\|y\|_{L^{p_{1}, q_{1}(\mathcal{M})}} .
$$

In particular, if $p=q=1$,

$$
|\tau(x y)| \leq\|x y\|_{L^{1}(\mathcal{M})} \leq\|x\|_{L^{p_{0}, q_{0}(\mathcal{M})}}\|y\|_{L^{p_{1}, q_{1}}(\mathcal{M})}, \forall x \in L^{p_{0}, q_{0}}(\mathcal{M}), y \in L^{p_{1}, q_{1}}(\mathcal{M}) .
$$

For $1<p<\infty, 1 \leq q<\infty$, this defines a natural duality

$$
\left(L^{p, q}(\mathcal{M})\right)^{*}=L^{p^{\prime}, q^{\prime}}(\mathcal{M})
$$

where $p^{\prime}, q^{\prime}$ denote the conjugate indices of $p, q$, respectively, and $\langle x, y\rangle=\tau(x y)$.

Let $\left(\mathcal{M}_{n}\right)_{n \geq 1}$ be an increasing sequence of von Neumann subalgebras of $\mathcal{M}$ such that the union of the $\mathcal{M}_{n}$ is weak*-dense in $\mathcal{M}$. For each $n \geq 1$, it is well known that there is unique normal faithful conditional expectation $\mathscr{E}_{n}$ from $\mathcal{M}$ onto $\mathcal{M}_{n}$. Moreover, $\mathscr{E}_{n}$ extends to a bounded projection from $L^{p, q}(\mathcal{M})$ onto $L^{p, q}\left(\mathcal{M}_{n}\right)$ for $1<p<\infty, 1 \leq q \leq \infty$, which we still denote by $\mathscr{E}_{n}$. 
For $1 \leq p<\infty, 1 \leq q \leq \infty$, and a finite sequence $a=\left(a_{n}\right)_{n \geq 1}$ in $\mathcal{M}$, we define

$$
\begin{aligned}
& \|a\|_{L^{p, q}\left(\mathcal{M} ; \ell_{c}^{2}\right)}=\left\|\left(\sum_{n}\left|a_{n}\right|^{2}\right)^{1 / 2}\right\|_{L^{p, q}(\mathcal{M})}, \\
& \|a\|_{L^{p, q}\left(\mathcal{M} ; \ell_{r}^{2}\right)}=\left\|\left(\sum_{n}\left|a_{n}^{*}\right|^{2}\right)^{1 / 2}\right\|_{L^{p, q}(\mathcal{M})}
\end{aligned}
$$

and

$$
\begin{aligned}
\|a\|_{L^{p, q}\left(\mathcal{M}, \mathscr{E}_{n-1} ; \ell_{c}^{2}\right)} & =\left\|\left(\sum_{n} \mathscr{E}_{n-1}\left|a_{n}\right|^{2}\right)^{1 / 2}\right\|_{L^{p, q}(\mathcal{M})}, \\
\|a\|_{L^{p, q}\left(\mathcal{M}, \mathscr{E}_{n-1} ; \ell_{r}^{2}\right)} & =\left\|\left(\sum_{n} \mathscr{E}_{n-1}\left|a_{n}^{*}\right|^{2}\right)^{1 / 2}\right\|_{L^{p, q}(\mathcal{M})}
\end{aligned}
$$

Now, any finite sequence $a=\left(a_{n}\right)$ in $L^{p, q}(\mathcal{M})$ can be regarded as an element in $L^{p, q}\left(\mathcal{M} \otimes B\left(\ell^{2}\right)\right)$. Therefore, $\|\cdot\|_{L^{p, q}\left(\mathcal{M}, \ell_{c}^{2}\right)}$ defines a quasi-norm on the family of all finite sequences in $L^{p, q}(\mathcal{M})$. The corresponding completion is a quasi-Banach space, denoted by $L^{p, q}\left(\mathcal{M}, \ell_{c}^{2}\right)$ (if $q=\infty$, the completion should be taken in a certain weak topology). It is shown in 9 , that $\|\cdot\|_{L^{p}\left(\mathcal{M}, \mathscr{E}_{n-1} ; \ell_{c}^{2}\right)}$ is a quasi-norm. Similarly, we can show that $\|\cdot\|_{L^{p, q}\left(\mathcal{M}, \mathscr{E}_{n-1} ; \ell_{c}^{2}\right)}$ defines a quasi-norm on the family of all finite sequences in $L^{p, q}(\mathcal{M})$. The corresponding completion is a quasi-Banach space, denoted by $L^{p, q}\left(\mathcal{M}, \mathscr{E}_{n-1} ; \ell_{c}^{2}\right)$. There are analogous arguments for $L^{p, q}\left(\mathcal{M}, \ell_{r}^{2}\right)$ and $L^{p, q}\left(\mathcal{M}, \mathscr{E}_{n-1} ; \ell_{r}^{2}\right)$.

Recall that a noncommutative martingale with respect to the filtration $\left(\mathcal{M}_{n}\right)_{n \geq 1}$ is a sequence $x=\left(x_{n}\right)_{n \geq 1}$ in $L^{1}(\mathcal{M}, \tau)$ such that

$$
\mathscr{E}_{n}\left(x_{n+1}\right)=x_{n}, \quad \forall n \geq 1 .
$$

If additionally $x \in L^{p, q}(\mathcal{M})$ for some $1<p<\infty, 0<q \leq \infty$, then $x$ is called an $L^{p, q}(\mathcal{M})$-martingale. In this case, we set

$$
\|x\|_{L^{p, q}(\mathcal{M})}=\sup _{n \geq 1}\left\|x_{n}\right\|_{L^{p, q}(\mathcal{M})} .
$$

If $\|x\|_{L^{p, q}(\mathcal{M})}<\infty$, then $x$ is called a bounded $L^{p, q}(\mathcal{M})$-martingale. The difference sequence $d x=\left(d x_{n}\right)_{n \geq 1}$ is defined by $d x_{n}=x_{n}-x_{n-1}$ with the usual convention that $x_{0}=0$. For concrete natural examples of noncommutative martingales, we refer to [16].

We describe the square functions and conditional square functions of noncommutative martingales. Following [9, [10, and [12, we will consider the following column and row versions of the square function and conditional square function: for a finite martingale $x=\left(x_{n}\right)$, set (recalling that $\mathscr{E}_{0}=\mathscr{E}_{1}$ )

$$
\begin{gathered}
S_{c}(x)=\left(\sum_{n}\left|d x_{n}\right|^{2}\right)^{1 / 2}, \quad S_{r}(x)=\left(\sum_{n}\left|d x_{n}^{*}\right|^{2}\right)^{1 / 2} ; \\
s_{c}(x)=\left(\sum_{n} \mathscr{E}_{n-1}\left|d x_{n}\right|^{2}\right)^{1 / 2}, \quad s_{r}(x)=\left(\sum_{n} \mathscr{E}_{n-1}\left|d x_{n}^{*}\right|^{2}\right)^{1 / 2} .
\end{gathered}
$$

Observe that

$$
\left\|S_{c}(x)\right\|_{L^{p, q}(\mathcal{M})}=\|d x\|_{L^{p, q}\left(\mathcal{M} ; \ell_{c}^{2}\right)}, \quad\left\|s_{c}(x)\right\|_{L^{p, q}(\mathcal{M})}=\|d x\|_{L^{p, q}\left(\mathcal{M}, \mathscr{E}_{n-1} ; \ell_{c}^{2}\right)} .
$$


The following is the conditioned Hölder inequality for Lorentz spaces. Throughout this paper, the letter $C$ will denote a positive constant, depending only the indices under consideration, which may change from line to line.

Proposition 2.1. Let $0<p_{k}, q_{k} \leq \infty$ for $k=0,1$ and $1 / p=1 / p_{0}+1 / p_{1}, 1 / q=$ $1 / q_{0}+1 / q_{1}$. Then for any $\left(a_{n}\right) \in L^{p_{0}, q_{0}}\left(\mathcal{M}, \mathscr{E}_{n} ; l_{c}^{2}\right),\left(b_{n}\right) \in L^{p_{1}, q_{1}}\left(\mathcal{M}, \mathscr{E}_{n} ; l_{c}^{2}\right)$,

$$
\left\|\sum_{n} \mathscr{E}_{n}\left(a_{n}^{*} b_{n}\right)\right\|_{L^{p, q}(\mathcal{M})} \leq C\left\|\left(a_{n}\right)\right\|_{L^{p_{0}, q_{0}\left(\mathcal{M}, \mathscr{E}_{n} ; l_{c}^{2}\right)}}\left\|\left(b_{n}\right)\right\|_{L^{p_{1}, q_{1}}\left(\mathcal{M}, \mathscr{E}_{n} ; l_{c}^{2}\right)}
$$

Proof. Following Proposition 2.8 in [9], for each $n \in \mathbb{N}$, we fix the isomorphism

$$
u_{p, q}^{n}: \quad L^{p, q}\left(\mathcal{M}, \mathscr{E}_{n}\right) \rightarrow L^{p, q}\left(\mathcal{M}_{n} ; l_{c}^{2}\right)
$$

and define

$$
u_{p, q}: \quad L^{p, q}\left(\mathcal{M}, \mathscr{E}_{n} ; l_{c}^{2}\right) \rightarrow L^{p, q}\left(\mathcal{M}_{n} ; l_{c}^{2}\left(\mathbb{N}^{2}\right)\right) \hookrightarrow L^{p, q}\left(\mathcal{M}_{n} \otimes B\left(l_{c}^{2}\left(\mathbb{N}^{2}\right)\right)\right)
$$

by

$$
u_{p, q}\left[\left(a_{n}\right)\right]=\left(u_{p, q}^{n}\left(a_{n}\right)_{k n}\right) .
$$

Similarly to Proposition 2.15 in [9], we obtain that $u_{p, q}$ is an isometric embedding. For any finite sequence, we apply Proposition 2.8 in 9 to obtain

$$
\left(u_{p_{0}, q_{0}}\left[\left(a_{n}\right)\right]\right)^{*} u_{p_{1}, q_{1}}\left[\left(b_{n}\right)\right]=\sum_{n}\left(u_{p_{0}, q_{0}}^{n}\left[\left(a_{n}\right)\right]\right)^{*} u_{p_{1}, q_{1}}^{n}\left[\left(b_{n}\right)\right]=\sum_{n} \mathscr{E}_{n}\left(a_{n}^{*} b_{n}\right) .
$$

So by Hölder's inequality, we have

$$
\begin{aligned}
& \left\|\sum_{n} \mathscr{E}_{n}\left(a_{n}^{*} b_{n}\right)\right\|_{L^{p, q}(\mathcal{M})}=\left\|\sum_{n}\left(u_{p_{0}, q_{0}}^{n}\left[\left(a_{n}\right)\right]\right)^{*} u_{p_{1}, q_{1}}^{n}\left[\left(b_{n}\right)\right]\right\|_{L^{p, q}\left(\mathcal{M}_{n} ; l_{c}^{2}\left(\mathbb{N}^{2}\right)\right)} \\
& =\left\|\left(\begin{array}{cc}
\sum_{n}\left(u_{p_{0}, q_{0}}^{n}\left[\left(a_{n}\right)\right]\right)^{*} u_{p_{1}, q_{1}}^{n}\left[\left(b_{n}\right)\right] & 0 \\
0 & 0
\end{array}\right)\right\|_{L^{p, q}\left(\mathcal{M}_{n} \otimes B\left(l_{c}^{2}\left(\mathbb{N}^{2}\right)\right)\right)} \\
& \leq C\left\|\left(\sum_{n}\left(u_{p_{0}, q_{0}}^{n}\left[\left(a_{n}\right)\right]\right)^{*} u_{p_{0}, q_{0}}^{n}\left[\left(a_{n}\right)\right]\right)^{1 / 2}\right\|_{L^{p_{0}, q_{0}}\left(\mathcal{M}_{n} ; l_{c}^{2}\left(\mathbb{N}^{2}\right)\right)} \\
& \times\left\|\left(\sum_{n}\left(u_{p_{1}, q_{1}}^{n}\left[\left(b_{n}\right)\right]\right)^{*} u_{p_{1}, q_{1}}^{n}\left[\left(b_{n}\right)\right]\right)^{1 / 2}\right\|_{L^{p_{1}, q_{1}}\left(\mathcal{M}_{n} ; l_{c}^{2}\left(\mathbb{N}^{2}\right)\right)} \\
& =C\left\|u_{p_{0}, q_{0}}\left[\left(a_{n}\right)\right]\right\|_{L^{p_{0}, q_{0}\left(\mathcal{M}_{n} ; l_{c}^{2}\left(\mathbb{N}^{2}\right)\right)}}\left\|u_{p_{1}, q_{1}}\left[\left(b_{n}\right)\right]\right\|_{L^{p_{1}, q_{1}\left(\mathcal{M}_{n} ; l_{c}^{2}\left(\mathbb{N}^{2}\right)\right)}} \\
& \leq C\left\|\left(a_{n}\right)\right\|_{L^{p_{0}, q_{0}}\left(\mathcal{M}, \mathscr{E}_{n} ; l_{c}^{2}\right)}\left\|\left(b_{n}\right)\right\|_{L^{p_{1}, q_{1}}\left(\mathcal{M}, \mathscr{E}_{n} ; l_{c}^{2}\right)} \text {. }
\end{aligned}
$$

The proof is finished.

\section{Burkholder's inequalities}

We now investigate Burkholder's inequalities for noncommutative Lorentz spaces. The principal result of this section is the following:

Theorem 3.1. Let $1<p<\infty, 1 \leq q<\infty$ and let $x=\left(x_{n}\right)_{n \geq 1}$ be a finite $L^{p, q}(\mathcal{M})$-martingale. Then

(1) for $2<p<\infty$,

$$
\begin{aligned}
& \|x\|_{L^{p, q}(\mathcal{M})} \\
& \quad \approx \max \left\{\left\|\sum_{n} d x_{n} \otimes e_{n}\right\|_{L^{p, q}\left(\mathcal{M} \otimes \ell^{\infty}\right)},\left\|s_{c}(x)\right\|_{L^{p, q}(\mathcal{M})},\left\|s_{r}(x)\right\|_{L^{p, q}(\mathcal{M})}\right\}
\end{aligned}
$$


(2) for $1<p<2$,

$\|x\|_{L^{p, q}(\mathcal{M})}$

$$
\approx \inf _{x=y+z+w}\left\{\left\|\sum_{n} d y_{n} \otimes e_{n}\right\|_{L^{p, q}\left(\mathcal{M} \otimes \ell^{\infty}\right)}+\left\|s_{c}(z)\right\|_{L^{p, q}(\mathcal{M})}+\left\|s_{r}(w)\right\|_{L^{p, q}(\mathcal{M})}\right\},
$$

where the infimum runs over all decompositions $d x_{n}=d y_{n}+d z_{n}+d w_{n}$ with $d y_{n}, d z_{n}$, and $d w_{n}$ being martingale difference sequences.

We will employ the discrete version of the $J$-method. For $0<\theta<1$ and $1 \leq q<\infty$, we denote by $\lambda^{\theta, q}$ the space of all sequences $\left(\alpha_{m}\right)_{m=-\infty}^{\infty}$ for which

$$
\left\|\left(\alpha_{m}\right)\right\|_{\lambda^{\theta, q}}=\left(\sum_{m \in \mathbb{Z}}\left(2^{-m \theta}\left|\alpha_{m}\right|\right)^{q}\right)^{1 / q}<\infty .
$$

Let $\left(E_{0}, E_{1}\right)$ be a compatible pair and suppose that $0<\theta<1$ and $1 \leq q<\infty$. The interpolation space $\left(E_{0}, E_{1}\right)_{\theta, q ; J}$ consists of elements $x \in E_{0}+E_{1}$ which admit a representation

$$
\left.x=\sum_{m \in \mathbb{Z}} u_{m} \quad \text { (convergence in } \quad E_{0}+E_{1}\right)
$$

with $u_{m} \in E_{0} \cap E_{1}$ and are such that

$$
\|x\|_{\theta, q ; J}=\inf \left\{\left\|\left\{J\left(u_{m}, 2^{m}\right)\right\}\right\|_{\lambda^{\theta, q}}\right\}<\infty
$$

where the infimum is taken over all representations of $x$ as in (3.3).

The following lemma from [15] is the key ingredient of our proof.

Lemma 3.2. Let $x=\left(x_{n}\right)_{1 \leq n \leq N}$ be a finite $L^{2}$-martingale. Then there exist three adapted sequences $a=\left(a_{n}\right)_{1 \leq n \leq N}, b=\left(b_{n}\right)_{1 \leq n \leq N}$, and $c=\left(c_{n}\right)_{1 \leq n \leq N}$ in $L^{2}(\mathcal{M}, \tau)$ such that:

(1) for every $1 \leq n \leq N$, we have the decomposition

$$
d x_{n}=a_{n}+b_{n}+c_{n}
$$

(2) the $L^{2}$-norms satisfy

$$
\|a\|_{L^{2}\left(\mathcal{M}, \ell_{c}^{2}\right)}+\|b\|_{L^{2}\left(\mathcal{M}, \ell_{c}^{2}\right)}+\|c\|_{L^{2}\left(\mathcal{M}, \ell_{r}^{2}\right)} \leq K\|x\|_{L^{2}(\mathcal{M})} ;
$$

(3) the conditional square functions satisfy the weak-type $(1,1)$ inequality

$$
\begin{aligned}
\left\|\sum_{n} a_{n} \otimes e_{n}\right\|_{L^{1, \infty}\left(\mathcal{M} \otimes \ell^{\infty}\right)} & +\left\|\left(\sum_{n} \mathscr{E}_{n-1}\left|b_{n}\right|^{2}\right)^{1 / 2}\right\|_{L^{1, \infty}(\mathcal{M})} \\
& +\left\|\left(\sum_{n} \mathscr{E}_{n-1}\left|c_{n}^{*}\right|^{2}\right)^{1 / 2}\right\|_{L^{1, \infty}(\mathcal{M})} \leq K\|x\|_{L^{1}(\mathcal{M})}
\end{aligned}
$$

where $\left(e_{n}\right)$ denotes the canonical unit of $\ell^{\infty}$ and $K$ is an absolute constant.

Proof of Theorem 3.1 .

Step 1. We first combine Lemma 3.2 and the $J$-method to prove the lower estimate of (3.2). Let $x=\left(x_{n}\right)_{1 \leq n \leq N}$ be any finite $L^{p, q}$-martingale. For $1<p<2$, we choose $\theta$ satisfying $1 / p=(1-\theta)+\theta / 2$. Fix $\left(u_{m}\right)_{m=-\infty}^{\infty}$ in $L^{2}(\mathcal{M})$ such that

$$
x_{N}=\sum_{m \in \mathbb{Z}} u_{m}
$$


and

$$
\left\|\left\{J\left(u_{m}, 2^{m}\right)\right\}\right\|_{\lambda^{\theta, q}} \leq 2\left\|x_{N}\right\|_{\theta, q ; J}
$$

where the $J$-functional and the interpolation are relative to the $\left(L^{1}(\mathcal{M}), L^{2}(\mathcal{M})\right)$ couple. By Lemma 3.2, for each fixed $m \in \mathbb{Z}$, we can find three finite adapted sequences $a^{m}, b^{m}$, and $c^{m}$ in $L^{2}(\mathcal{M})$ and an absolute constant $K>0$ such that:

(1) $\mathscr{E}_{n}\left(u_{m}\right)-\mathscr{E}_{n-1}\left(u_{m}\right)=a_{n}^{m}+b_{n}^{m}+c_{n}^{m}$ for all $1 \leq n \leq N$;

(2) $J\left(\sum_{n \geq 1} a_{n}^{m} \otimes e_{n}, t ; L^{1, \infty}\left(\mathcal{M} \otimes \ell^{\infty}\right), L^{2}\left(\mathcal{M} \otimes \ell^{\infty}\right)\right) \leq K J\left(u_{m}, t\right)$ for every $t>0$

(3) $J\left(\left(\sum_{n \geq 1} \mathscr{E}_{n-1}\left|b_{n}^{m}\right|^{2}\right)^{1 / 2}, t ; L^{1, \infty}(\mathcal{M}), L^{2}(\mathcal{M})\right) \leq K J\left(u_{m}, t\right)$ for every $t>$ 0

(4) $J\left(\left(\sum_{n \geq 1} \mathscr{E}_{n-1}\left|c_{n}^{m *}\right|^{2}\right)^{1 / 2}, t ; L^{1, \infty}(\mathcal{M}), L^{2}(\mathcal{M})\right) \leq K J\left(u_{m}, t\right)$ for every $t>$ 0 .

Then we deduce that

$$
\begin{gathered}
\left\|\left\{J\left(\sum_{n \geq 1} a_{n}^{m} \otimes e_{n}, 2^{m}\right)\right\}\right\|_{\lambda^{\theta, q}} \leq 2 K\left(\left\|x_{N}\right\|_{\theta, q ; J}\right), \\
\left\|\left\{J\left(\left(\sum_{n \geq 1} \mathscr{E}_{n-1}\left|b_{n}^{m}\right|^{2}\right)^{1 / 2}, 2^{m}\right)\right\}\right\|_{\lambda^{\theta, q}} \leq 2 K\left\|x_{N}\right\|_{\theta, q ; J},
\end{gathered}
$$

and

$$
\left\|\left\{J\left(\left(\sum_{n \geq 1} \mathscr{E}_{n-1}\left|c_{n}^{m *}\right|^{2}\right)^{1 / 2}, 2^{m}\right)\right\}\right\|_{\lambda^{\theta, q}} \leq 2 K\left\|x_{N}\right\|_{\theta, q ; J} .
$$

From (3.4) and the definition of $\|\cdot\|_{\theta, q ; J}$, we get that for any finite subset $S \subset \mathbb{Z}$,

$$
\begin{aligned}
& \left.\left\|\sum_{m \in S} \sum_{n \geq 1} a_{n}^{m} \otimes e_{n}\right\|_{\left[L^{1, \infty}\left(\mathcal{M} \otimes \ell^{\infty}\right), L^{2}(\mathcal{M} \otimes l \infty)\right.}\right]_{\theta, q ; J} \\
& \leq\left\|\left\{J\left(\sum_{n \geq 1} a_{n}^{m} \otimes e_{n}, 2^{m}\right)\right\}\right\|_{\lambda^{\theta, q}} \leq 2 K\left\|x_{N}\right\|_{\theta, q ; J}
\end{aligned}
$$

For fixed $m \in S, \alpha^{m}=\sum_{n \geq 1} a_{n}^{m} \otimes e_{n}$ is an element of the Banach space $\left[L^{1, \infty}(\mathcal{M} \otimes\right.$ $\left.\left.\ell^{\infty}\right), L^{2}\left(\mathcal{M} \otimes \ell^{\infty}\right)\right]_{\theta, q ; J}$. Then

$$
\left\|\sum_{m \in S} \pm \alpha^{m}\right\|_{\left[L^{1, \infty}\left(\mathcal{M} \otimes \ell^{\infty}\right), L^{2}\left(\mathcal{M} \otimes \ell^{\infty}\right)\right]_{\theta, q ; J}} \leq 4 K\left\|x_{N}\right\|_{\theta, q ; J} .
$$

This means (since the constant $C$ is independent of the finite subset of $\mathbb{Z}$ ) that the formal series $\sum_{m \in \mathbb{Z}} \alpha^{m}$ is weak unconditionally Cauchy (see for instance Diestel [6. p. 44, Theorem 6]); but since for $1 \leq q<\infty$ the Banach space $\left[L^{1, \infty}(\mathcal{M} \otimes\right.$ $\left.\left.\ell^{\infty}\right), L^{2}\left(\mathcal{M} \otimes \ell^{\infty}\right)\right]_{\theta, q ; J}$ contains no copy of $c_{0}$, this implies that the series $\sum_{m \in \mathbb{Z}} \alpha^{m}$ is (unconditionally) convergent. Hence if we set

$$
a:=\sum_{m \in \mathbb{Z}} \alpha^{m}
$$

then the sequence $a=\left(a_{n}\right)_{n \geq 1}$ satisfies

$$
\left\|\sum_{n \geq 1} a_{n} \otimes e_{n}\right\|_{\left[L^{1, \infty}\left(\mathcal{M} \otimes \ell^{\infty}\right), L^{2}\left(\mathcal{M} \otimes \ell^{\infty}\right)\right]_{\theta, q ; J}} \leq 2 K\left\|x_{N}\right\|_{\theta, q ; J}
$$


Now we consider the space $L^{2}\left(\mathcal{M}, \mathscr{E}_{n-1} ; \ell_{c}^{2}\right)$ as the column subspace of $L^{2}(\mathcal{M} \otimes$ $\left.B\left(\ell^{2}\left(\mathbb{N}^{2}\right)\right)\right)$ and view the sequence $b^{m}=\left(b_{n}^{m}\right)_{n \geq 1}$ as a column vector with entries from $L^{2}\left(\mathcal{M} \otimes B\left(\ell^{2}\left(\mathbb{N}^{2}\right)\right)\right.$ ) (see [9] for more details). Then for any fixed $m \in \mathbb{Z}$,

$$
\begin{aligned}
& J\left(\left(\sum_{n \geq 1} \mathscr{E}_{n-1}\left|b_{n}^{m}\right|^{2}\right)^{1 / 2}, 2^{m}\right) \\
& \quad=\max \left\{\left\|\left(\sum_{n \geq 1} \mathscr{E}_{n-1}\left|b_{n}^{m}\right|^{2}\right)^{1 / 2}\right\|_{L^{1, \infty}(\mathcal{M})}, 2^{m}\left\|\left(\sum_{n \geq 1} \mathscr{E}_{n-1}\left|b_{n}^{m}\right|^{2}\right)^{1 / 2}\right\|_{L^{2}(\mathcal{M})}\right\} \\
& \quad=J\left(b^{m}, 2^{m} ; L^{1, \infty}\left(\mathcal{M} \otimes B\left(\ell^{2}\left(\mathbb{N}^{2}\right)\right)\right), L^{2}\left(\mathcal{M} \otimes B\left(\ell^{2}\left(\mathbb{N}^{2}\right)\right)\right)\right) .
\end{aligned}
$$

Then (3.5) becomes

$$
\left\|\left\{J\left(b^{m}, 2^{m} ; L^{1, \infty}\left(\mathcal{M} \otimes B\left(\ell^{2}\left(\mathbb{N}^{2}\right)\right)\right), L^{2}\left(\mathcal{M} \otimes B\left(\ell^{2}\left(\mathbb{N}^{2}\right)\right)\right)\right)\right\}\right\|_{\lambda^{\theta, q}} \leq 2 K\left\|x_{N}\right\|_{\theta, q ; J} .
$$

Similarly, if we set $b:=\sum_{m \in \mathbb{Z}} b^{m}$, then $b=\left(b_{n}\right)_{n \geq 1}$ as a column vector satisfies

$$
\left.\|b\|_{\left[L^{1, \infty}\left(\mathcal{M} \otimes B\left(\ell^{2}\left(\mathbb{N}^{2}\right)\right)\right), L^{2}\left(\mathcal{M} \otimes B\left(\ell^{2}\left(\mathbb{N}^{2}\right)\right)\right)\right.}\right]_{\theta, q ; J} \leq 2 K\left\|x_{N}\right\|_{\theta, q ; J} .
$$

Again, upon setting $c:=\sum_{m \in \mathbb{Z}} c^{m}$, we have

$$
\left.\|c\|_{\left[L^{1, \infty}\left(\mathcal{M} \otimes B\left(\ell^{2}\left(\mathbb{N}^{2}\right)\right)\right), L^{2}\left(\mathcal{M} \otimes B\left(\ell^{2}\left(\mathbb{N}^{2}\right)\right)\right)\right.}\right]_{\theta, q ; J} \leq 2 K\left\|x_{N}\right\|_{\theta, q ; J}
$$

Note that $a, b$, and $c$ are adapted sequences. Moreover, it is clear from the construction that for $1 \leq n \leq N$,

$$
d x_{n}=a_{n}+b_{n}+c_{n} .
$$

Now we use the following well-known equalities: for $1 / p=(1-\theta)+\theta / 2,1 \leq q<\infty$, and any semifinite von Neumann algebra $\mathcal{N}$

$$
\left[L^{1, \infty}(\mathcal{N}), L^{2}(\mathcal{N})\right]_{\theta, q ; J}=L^{p, q}(\mathcal{N}) \quad \text { and } \quad\left[L^{1}(\mathcal{N}), L^{2}(\mathcal{N})\right]_{\theta, q ; J}=L^{p, q}(\mathcal{N}) .
$$

Combining the previous inequalities, we conclude that there is a positive constant $C>0$ such that

$$
\begin{aligned}
\left\|\sum_{n \geq 1} a_{n} \otimes e_{n}\right\|_{L^{p, q}\left(\mathcal{M} \otimes \ell^{\infty}\right)} & +\left\|\left(\sum_{n \geq 1} \mathscr{E}_{n-1}\left|b_{n}\right|^{2}\right)^{1 / 2}\right\|_{L^{p, q}(\mathcal{M})} \\
& +\left\|\left(\sum_{n \geq 1} \mathscr{E}_{n-1}\left|c_{n}^{*}\right|^{2}\right)^{1 / 2}\right\|_{L^{p, q}(\mathcal{M})} \leq C\|x\|_{L^{p, q}(\mathcal{M})} .
\end{aligned}
$$

To complete the proof, it is enough to set, for $n \geq 1$,

$$
d y_{n}=a_{n}-\mathscr{E}_{n-1}\left(a_{n}\right), \quad d z_{n}=b_{n}-\mathscr{E}_{n-1}\left(b_{n}\right), \quad d w_{n}=c_{n}-\mathscr{E}_{n-1}\left(c_{n}\right) .
$$

Then $\left(d y_{n}\right)_{\geq 1},\left(d z_{n}\right)_{\geq 1}$, and $\left(d w_{n}\right)_{\geq 1}$ are martingale difference sequences with $d x_{n}=$ $d y_{n}+d z_{n}+d w_{n}$. Note that since $\mathscr{E}_{n-1}$ is bounded in $L^{p}(\mathcal{M})$, by interpolation we have that for $1<p<\infty, 1 \leq q<\infty$,

So

$$
\left\|\sum \mathscr{E}_{n-1}\left(a_{n}\right) \otimes e_{n}\right\|_{L^{p, q}\left(\mathcal{M} \otimes \ell^{\infty}\right)} \leq C\left\|\sum a_{n} \otimes e_{n}\right\|_{L^{p, q}\left(\mathcal{M} \otimes \ell^{\infty}\right)} .
$$

$$
\left\|\sum_{n} d y_{n} \otimes e_{n}\right\|_{L^{p, q}\left(\mathcal{M} \otimes \ell^{\infty}\right)} \leq C\left\|\sum a_{n} \otimes e_{n}\right\|_{L^{p, q}\left(\mathcal{M} \otimes \ell^{\infty}\right)} \leq C\|x\|_{L^{p, q}(\mathcal{M})} .
$$

Noting that $\mathscr{E}_{n-1}\left(b_{n}\right)^{*} \mathscr{E}_{n-1}\left(b_{n}\right) \leq \mathscr{E}_{n-1}\left(b_{n}^{*} b_{n}\right)$, we have

$$
\mathscr{E}_{n-1}\left|d z_{n}\right|^{2}=\mathscr{E}_{n-1}\left(b_{n}^{*} b_{n}\right)-\mathscr{E}_{n-1}\left(b_{n}\right)^{*} \mathscr{E}_{n-1}\left(b_{n}\right) \leq \mathscr{E}_{n-1}\left(b_{n}^{*} b_{n}\right) .
$$


Then we finally deduce that

$$
\left\|\sum_{n} d y_{n} \otimes e_{n}\right\|_{L^{p, q}\left(\mathcal{M} \otimes \ell^{\infty}\right)}+\left\|s_{c}(z)\right\|_{L^{p, q}(\mathcal{M})}+\left\|s_{r}(w)\right\|_{L^{p, q}(\mathcal{M})} \leq C\|x\|_{L^{p, q}(\mathcal{M})} .
$$

Step 2. Applying the inequality established in Step 1, by duality we now prove the upper estimate of (3.1). Let $x=\left(x_{n}\right)_{n \geq 1}$ be any finite martingale, say $x_{n}=x_{N}$ for all $n \geq N$. For $2<p<\infty$ and $1 \leq q<\infty$, let $b_{N} \in L^{p^{\prime}, q^{\prime}}(\mathcal{M}), 1<p^{\prime}<2,1<$ $q^{\prime} \leq \infty$. Then $b_{N}$ defines a finite martingale $b=\left(b_{n}\right)_{n \geq 1}$,

$$
b_{n}=\mathscr{E}_{n}\left(b_{N}\right), \quad n \geq 1
$$

Let $b_{n}=y_{n}+w_{n}+z_{n}$ be any decomposition of $b$ satisfying the conditions in Lemma 3.2. We then obtain by Hölder's inequality and Proposition 2.1 that

$$
\begin{aligned}
\left|\tau\left(x_{N}^{*} b_{N}\right)\right|= & \left|\tau\left(\sum_{n} d x_{n}^{*} d b_{n}\right)\right| \\
\leq & \left|\tau\left(\sum_{n} d x_{n}^{*} d y_{n}\right)\right|+\left|\tau\left(\sum_{n} d x_{n}^{*} d w_{n}\right)\right|+\left|\tau\left(\sum_{n} d x_{n}^{*} d z_{n}\right)\right| \\
= & \left|\tau \otimes \operatorname{tr}\left(\sum_{n}\left(d x_{n}^{*} \otimes e_{n}\right)\left(d y_{n} \otimes e_{n}\right)\right)\right| \\
& +\left|\tau\left(\sum_{n} \mathscr{E}_{n-1} d x_{n}^{*} d w_{n}\right)\right|+\left|\tau\left(\sum_{n} \mathscr{E}_{n-1} d x_{n}^{*} d z_{n}\right)\right| \\
\leq & \left\|\sum_{n} d x_{n}^{*} \otimes e_{n}\right\|_{L^{p, q}\left(\mathcal{M} \otimes \ell^{\infty}\right)}\left\|\sum_{n} d y_{n} \otimes e_{n}\right\|_{L^{p^{\prime}, q^{\prime}}\left(\mathcal{M} \otimes \ell^{\infty}\right)} \\
& +\left\|s_{c}(x)\right\|_{L^{p, q}(\mathcal{M})}\left\|s_{c}(w)\right\|_{L^{p^{\prime}, q^{\prime}}(\mathcal{M})}+\left\|s_{r}(x)\right\|_{L^{p, q}(\mathcal{M})}\left\|s_{r}(z)\right\|_{L^{p^{\prime}, q^{\prime}}(\mathcal{M})} \\
\leq & C \max \left\{\left\|\sum_{n} d x_{n} \otimes e_{n}\right\|_{L^{p, q}\left(\mathcal{M} \otimes \ell^{\infty}\right)},\right. \\
& \left.\left\|s_{c}(x)\right\|_{L^{p, q}(\mathcal{M})},\left\|s_{r}(x)\right\|_{L^{p, q}(\mathcal{M})}\right\}\left\|b_{N}\right\|_{L^{p^{\prime}, q^{\prime}}(\mathcal{M})} .
\end{aligned}
$$

Taking the supremum over all $b_{N}$ such that $\left\|b_{N}\right\|_{L^{p^{\prime}, q^{\prime}}(\mathcal{M})} \leq 1$, we deduce that $\|x\|_{L^{p, q}(\mathcal{M})} \leq C \max \left\{\left\|\sum_{n} d x_{n} \otimes e_{n}\right\|_{L^{p, q}\left(\mathcal{M} \otimes l^{\infty}\right)},\left\|s_{C}(x)\right\|_{L^{p, q}(\mathcal{M})},\left\|s_{r}(x)\right\|_{L^{p, q}(\mathcal{M})}\right\}$.

So we obtain the desired result.

Step 3. Now we prove the lower estimate of (3.1). First, we observe that

$$
\left\|\sum_{n} d x_{n} \otimes e_{n}\right\|_{L^{2}\left(\mathcal{M} \otimes \ell^{\infty}\right)} \leq\|x\|_{L^{2}(\mathcal{M})}, \quad\left\|\sum_{n} d x_{n} \otimes e_{n}\right\|_{L^{\infty}\left(\mathcal{M} \otimes \ell^{\infty}\right)} \leq\|x\|_{L^{\infty}(\mathcal{M})} .
$$

Then by interpolation we get that for $2<p<\infty, 0<q \leq \infty$,

$$
\left\|\sum_{n} d x_{n} \otimes e_{n}\right\|_{L^{p, q}\left(\mathcal{M} \otimes \ell^{\infty}\right)} \leq C\|x\|_{L^{p, q}(\mathcal{M})} .
$$

Thus it remains to majorize $\left\|s_{c}(x)\right\|_{L^{p, q}(\mathcal{M})}$ and $\left\|s_{r}(x)\right\|_{L^{p, q}(\mathcal{M})}$. Again, we view $L^{p}\left(\mathcal{M} ; \mathscr{E}_{n-1}, \ell_{c}^{2}\right)$ as a closed subspace of $L^{p}\left(\mathcal{M} \otimes B\left(\ell^{2}\left(\mathbb{N}^{2}\right)\right)\right)$. Then there exists a linear operator $T$ such that

$$
\left\|s_{c}(x)\right\|_{L^{p}(\mathcal{M})}=\left\|\left(d x_{n}\right)\right\|_{L^{p}\left(\mathcal{M} ; \mathscr{E}_{n-1}, \ell_{c}^{2}\right)}=\left\|T\left(d x_{n}\right)\right\|_{L^{p}\left(\mathcal{M} \otimes B\left(\ell^{2}\left(\mathbb{N}^{2}\right)\right)\right)} .
$$


From Theorem 6.1 in [10, we know that for any $2 \leq r<\infty$,

$$
\left\|T\left(d x_{n}\right)\right\|_{L^{r}\left(\mathcal{M} \otimes B\left(\ell^{2}\left(\mathbb{N}^{2}\right)\right)\right)} \leq C\|x\|_{L^{r}(\mathcal{M})} .
$$

By interpolation,

$$
\left\|T\left(d x_{n}\right)\right\|_{L^{p, q}\left(\mathcal{M} \otimes B\left(\ell^{2}\left(\mathbb{N}^{2}\right)\right)\right)} \leq C\|x\|_{L^{p, q}(\mathcal{M})} .
$$

Thus we obtain

$$
\left\|s_{c}(x)\right\|_{L^{p, q}(\mathcal{M})} \leq C\|x\|_{L^{p, q}(\mathcal{M})} .
$$

The same argument can also be applied to $\left\|s_{r}(x)\right\|_{L^{p, q}(\mathcal{M})}$, and we obtain the desired inequality.

Step 4. The proof of the lower estimate of (3.2) is similar to Step 2, using the result in Step 3; therefore we omit it. Thus the proof of Theorem 3.1 is complete.

Remark 3.3. In the same spirit as Theorem 3.1, we can extend the BurkholderGundy inequalities of Pisier and Xu [13] to Lorentz spaces, too.

\section{ACKNOWLEDGEMENTS}

The author would like to thank the referee and the editor for their helpful comments.

\section{REFERENCES}

[1] D. L. Burkholder. Distribution function inequalities for martingales. Ann. Probab., 1:19-42, 1973. MR0365692 (51:1944)

[2] D. L. Burkholder, R. F. Gundy. Extrapolation and interpolation of quasi-linear operators on martingales. Acta. Math., 124:249-304, 1970. MR.0440695 (55:13567)

[3] J. Bergh and J. Löfström. Interpolation spaces: an introduction. Berlin, Heidelberg, New York: Springer, 1976. MR0482275 (58:2349)

[4] C. Bennett, R. Sharpley. Interpolation of operators. Academic Press, 1988. MR0928802 (89e:46001)

[5] N. L. Carothers, S. J. Dilworth. Inequalities for sums of independent random variables. Proc. Amer. Math. Soc., 104:221-226, 1988. MR958071 (90f:60005)

[6] J. Diestel. Sequences and series in Banach spaces. New York, Berlin, Tokyo: Springer, 1984. MR.737004 (85i:46020)

[7] R. Hunt. On $L^{p, q}$ spaces. L'Ens. Math., 12:249-275, 1966. MR0223874 (36:6921)

[8] R. O'Neil. Convolution operators, and $L^{p, q}$ spaces. Duke. Math. J., 30:129-143, 1963. MR0146673 (26:4193)

[9] M. Junge. Doob's inequality for noncommutative martingales. J. Reine Angew. Math., 549:149-190, 2002. MR1916654 (2003k:46097)

[10] M. Junge, Q. Xu. Noncommutative Burkholder/Rosenthal inequalities. Ann. Probab., 31:948995, 2003. MR.1964955(2004f:46078)

[11] M. Junge, Q. Xu. Noncommutative Burkholder/Rosenthal inequalities. II: Applications. Israel J. Math., 167(1):227-282, 2008. MR2448025

[12] G. Pisier, Q. Xu. Noncommutative martingale inequalities. Comm. Math. Phys., 189:667-698, 1997. MR 1482934 (98m:46079)

[13] G. Pisier, Q. Xu. Noncommutative $L^{p}$ spaces. Handbook of the geometry of Banach spaces, Vol. 2, 1459-1517. Amsterdam: Elsevier, 2003. MR 1999201(2004i:46095)

[14] H. P. Rosenthal. On the subspaces of $L_{p}(p>2)$ spanned by sequences of independent random variables. Israel J. Math., 8:273-303, 1970. MR0271721(42:6602) 
[15] N. Randrianantoanina. Conditional square functions for noncommutative martingales. Ann. Probab., 35:1039-1070, 2007. MR2319715 (2009d:46112)

[16] Q. Xu. Recent development on noncommutative martingale inequalities. Functional space theory and its applications, Proceedings of International Conference in China, Wuhan, 2003, 283-314.

Institute of Probability and Statistics, Central South University, Changsha 410075, People's Republic of China - and - Laboratoire de Mathématiques, Université de Franche-Comté, 25030 Besançon Cedex, France

Current address: Institute of Probability and Statistics, Central South University, Changsha 410075, People's Republic of China

E-mail address: jjiao@univ-fcomte.fr 